\title{
Dysregulated IncRNA TUG1 in different pulmonary artery cells under hypoxia
}

\author{
Zhenchun Lv ${ }^{1,2 \#}$, Rong Jiang ${ }^{1 \#}$, Xiaoyi Hu${ }^{1,3 \#}$, Qinhua Zhao ${ }^{1}$, Yuanyuan Sun ${ }^{1}$, Lan Wang ${ }^{1}$, Jinling Li $^{1}$, \\ Yuqing $\mathrm{Miao}^{2}$, Wenhui $\mathrm{Wu}^{1}$, Ping Yuan ${ }^{1}$ \\ ${ }^{1}$ Department of Cardio-Pulmonary Circulation, Shanghai Pulmonary Hospital, Tongji University, School of Medicine, Shanghai, China; ${ }^{2}$ Institute \\ of Bismuth Science, University of Shanghai for Science and Technology, Shanghai, China; ${ }^{3}$ Department of Gastroenterology, the 903rd Hospital of \\ People's Liberation Army, Hangzhou, China \\ Contributions: (I) Conception and design: P Yuan, W Wu, Y Miao; (II) Administrative support: None; (III) Provision of study materials or patients: \\ None; (IV) Collection and assembly of data: Z Lv, R Jiang, Y Sun, L Wang, J Li, W Wu, P Yuan; (V) Data analysis and interpretation: Z Lv, W Wu, \\ P Yuan; (VI) Manuscript writing: All authors; (VII) Final approval of manuscript: All authors. \\ \#These authors contributed equally to this work. \\ Correspondence to: Ping Yuan, MD; Wenhui Wu, MD. Department of Cardio-Pulmonary Circulation, Shanghai Pulmonary Hospital, Tongji \\ University, School of Medicine, No. 507 Zhengmin Road, Shanghai 200433, China. Email: pandyyuan@tongji.edu.cn; wenhui5621006@126.com; \\ Yuqing Miao, MD. Department of Institute of Bismuth Science, University of Shanghai for Science and Technology, Shanghai 200093, China. \\ Email: yqmiao@ymail.com.
}

Background: At present, the role of lncRNAs in the pathogenesis of hypoxia-induced pulmonary hypertension (HPH) is not fully understood. This study aimed to explore differences in the hypoxia-induced expression of lncRNAs and their potential role in multiple pulmonary artery cells.

Methods: LncRNA expression in pulmonary artery smooth muscle cells (PASMCs), pulmonary microvascular endothelial cells (PMECs), and pericytes (PCs) was analyzed by high-throughput sequencing and compared between normoxic and hypoxic cells. Bioinformatics analysis was conducted to predict their functions.

Results: PASMCs, PMECs, and PCs displayed 275 (140 upregulated), 251 (162 upregulated), and 290 (176 upregulated) different lncRNAs, respectively. Among these, lncRNA TUG1 levels increased in PASMCs and PCs but decreased in PMECs. Bioinformatics analysis indicated that lncRNA TUG1 might target miR-145-5p, thereby affecting SOX4 and BMF expression, and could also regulate miR-129-5p levels to affect CYP1B1 and VCP expression. It could also regulate miR-138-5p levels to affect KCNK3 and RHOC expression.

Conclusions: Hypoxia exposure of vascular cells resulted in differential expression of lncRNAs, especially lncRNA TUG1, which showed significant abnormal expression in all three types of vascular cells under hypoxia. Our results suggested that abnormal expression of lncRNA TUG1 might be involved in the regulation of pulmonary vascular cell function under hypoxia.

Keywords: Pulmonary hypertension (PH); hypoxia; lncRNAs; pericytes

Submitted Apr 02, 2021. Accepted for publication May 19, 2021.

doi: 10.21037/atm-21-2040

View this article at: http://dx.doi.org/10.21037/atm-21-2040 


\section{Introduction}

Hypoxia causes vascular endothelial cell damage, which in turn perturbs the synthesis and secretion of various vasodilator factors and leads to pulmonary vasoconstriction in the short term and pulmonary vascular remodeling in the longer term (1). Such vascular remodeling is characterized by thickening of the medial membrane due to proliferation of phenotypically altered smooth muscle cells, and endothelial cell dysfunction not only leads to intimal thickening and distal pulmonary artery dilatation, but also inflammation and infiltration of the outer membrane (2). In addition, some studies have reported that pericytes (PCs), a layer of cells present outside certain small pulmonary vascular endothelial cells, are also involved in the process of pulmonary vascular remodeling in pulmonary hypertension (PH) (3). To date, phenotypic changes in PCs during hypoxia-induced $\mathrm{PH}(\mathrm{HPH})$ have been rarely studied, and while there have been many studies on the epigenetic mechanisms of phenotypic changes in pulmonary vascular cells, the role of long non-coding RNAs (lncRNAs) and their possible abnormal expression in the various parietal cells involved in HPH remain unclear (4).

LncRNAs are roughly defined as non-coding RNA molecules with a transcript length of more than $200 \mathrm{nt}$ that lack protein-coding ability but nevertheless function as RNAs (5). LncRNAs were originally considered to be "transcriptional noise", however, they are now known to be biologically active molecules involved in important mechanisms that regulate gene expression. Notably, lncRNAs are predominantly found in vascular cells and are correlated with vascular functions (6). One study demonstrated that the proliferation and apoptosis of vascular smooth muscle cells were regulated by lncRNAs (7). Another study has confirmed that the lncRNA NONHSAT073641 can promote the proliferation of vascular endothelial cells (8). However, lncRNA expression profiles in various pulmonary artery cells under hypoxic conditions, and particularly in PCs, have not been reported.

Therefore, in this study, we comprehensively described and compared abnormalities in lncRNA expression profiles of pulmonary artery smooth muscle cells (PASMCs), pulmonary microvascular endothelial cells (PMECs), and PCs under normoxic and hypoxic conditions using high-throughput sequencing technology. In addition, bioinformatics analysis, including Gene Ontology (GO) analysis, Kyoto Encyclopedia of Genes and Genomes (KEGG) pathway analysis, and the prediction of lncRNA-
miRNA mechanisms of action, were performed to provide basic data for future studies on the role of lncRNAs in the pathogenesis of $\mathrm{PH}$. We present the following article in accordance with the MDAR reporting checklist (available at http://dx.doi.org/10.21037/atm-21-2040).

\section{Methods}

All procedures followed were in accordance with the ethical standards of the responsible committee on human experimentation (institutional and national) and with the Helsinki Declaration (as revised in 2013). Shanghai Pulmonary Hospital reviewed and approved the protocol form prior to initiation of the study (approval number, K20195Y).

\section{Cell culture}

Human PASMCs, PMECs, and PCs were acquired from Science Cell (Shanghai, China) and were cultured in smooth muscle cell medium, endothelial cell culture medium, and pericyte medium containing $4 \mathrm{mM} \mathrm{L}$-glutamine and $10 \%$ fetal bovine serum, respectively. Cells were cultured under normoxia and hypoxia in a humidified incubator. The condition of normoxia was $37^{\circ} \mathrm{C}$ with a $95 \%$ air, $5 \% \mathrm{CO}_{2}$ mixture. Hypoxia was induced in PASMCs, PMECs, and PCs by incubating them in a gas mixture containing $92 \%$ $\mathrm{N}_{2}, 5 \% \mathrm{CO}_{2}$, and $3 \% \mathrm{O}_{2}$ for $24 \mathrm{~h}$. Before each experiment, cells were starved for $6 \mathrm{~h}$ in serum-free medium.

\section{Whole transcriptome sequencing of lncRNAs and bioinformatics analysis}

Total RNA was extracted from three paired different type cells exposed to normoxic and hypoxic conditions using the TRIzol reagent (Invitrogen, CA, USA), according to the manufacturer's protocol.

Based on the detail of manufacturer's instructions, TruSeq Stranded Total RNA with Ribo-Zero Gold (Illumina, CA, USA) were used to build the three lncRNAs libraries. Sequenced were performed on Illumina HiSeqTM 4000 sequencing platform (CA, USA). Sequencing was performed by Shanghai OEbiotech Co. (Shanghai, China) (9).

All sequencing procedures and analyses were performed by OEbiotech (Shanghai, China) and differentially expressed genes were identified using DESeq software (10). The different expressions of mRNAs with the fold changes (FC) $\geq 2$ or $\leq 0.5$ and $\mathrm{P}<0.05$ were analyzed in three paired 
groups. The GO terms and KEGG pathways with $\mathrm{P}<0.05$ were considered significantly enriched.

\section{Real-time polymerase chain reaction}

Real-time polymerase chain reaction (PCR) was used to confirm lncRNA expression profile data derived using highthroughput sequencing. All sequencing procedures and validation of the analyses were performed by OEbiotech (Shanghai, China). Differentially expressed genes were identified using DESeq software. The primer sequences used were as follows: lncRNA LNCOG: Forward: 5 '-TTCAAATCGGGACCCCACTG-3', Reverse: 5'-TAGGGCCTTGTCCTTTGCTG-3'; IncRNA TUG1: Forward: 5'-GGCACCCAGTGTAAAGCA-3', Reverse: 5'-AAGCAGCAGATAACAGAGTTGA-3'.

\section{Statistical analyses}

Statistical analysis was performed using negative binomial distribution to compare high-throughput sequencing data from the two groups of samples. Differences with fold $\mathrm{FC} \geq 2$ or $\leq 0.5$ and $\mathrm{P}<0.05$ were considered statistically significant. The false discovery rate was calculated to correct the $\mathrm{P}$ value on analysis. For real-time PCR analysis, the results are the means of at least three independent experiments $\pm \mathrm{SE}$, and the expression level of each lncRNA was represented as FC using the $2^{-\Delta \Delta \mathrm{Ct}}$ method. P values $<0.05$ were considered statistically significant.

\section{Results}

\section{LncRNA sequencing and analysis}

To better understand the molecular aspects of the pathogenesis of $\mathrm{PH}$, we performed a comparative full transcriptomic analysis of three sets of normoxic and hypoxic PASMCs, PMECs, and PCs. Thus, we constructed 18 cDNA libraries, named PASMC_C1, PASMC_C2, PASMC_C3, PASMC_H1, PASMC_H2, PASMC_H3, PMEC_C1, PMEC_C2, PMEC_C3, PMEC_H1, PMEC_ H2, PMEC_H3, PC_C1, PC_C2, PC_C3, PC_H1, PC_ $\mathrm{H} 2$, and PC_H3.

\section{Volcano plot and clustered heat map of PASMCs, PMECs, and PCs}

The volcano plot and heatmap of lncRNAs in PASMCs,
PMECs, and PCs are provided in Figure 1. The volcano plot showed significant variation in lncRNA expression between both groups. Between normoxic and hypoxic PASMCs, 276 genes were differentially expressed, which corresponded to 140 upregulated genes and 136 downregulated genes. Among these, lncRNA XLOC_004748 (chr 12:2268584422700706) showed the highest upregulation, while lncRNA LOC105376095 (chr 9:76608665-76652293) was the most downregulated gene, with an 0.009-fold change. The most upregulated and downregulated lncRNAs in PASMCs are listed in Figure $1 A$.

A total of 251 genes were differentially expressed between normoxic and hypoxic PMECs, which included 162 upregulated genes and 89 downregulated genes. Among them, MIR210HG (chr 11:567144-568457), with a 573-fold change, was the most upregulated lncRNA, while MALAT1 (chr 11:65499042-65500970), with an 0.00028-fold change, was the most downregulated. The most upregulated and downregulated lncRNAs in PMECs are listed in Figure 1C.

In PCs, a total of 291 genes were differentially expressed between normoxic and hypoxic cells, including 177 upregulated genes and 114 downregulated genes. Among them, WARS2-IT1 (chr 1:119047405-119063287), with a 298-fold change, was the most upregulated lncRNA, while XLOC_001324 (chr 1:16512524-16677340), with an 0.007 -fold change, was the most downregulated. The most upregulated and downregulated lncRNAs in PCs are listed in Figure $1 E$.

\section{Categories of differentially expressed lncRNAs in PASMCs, PMECs, and PCs}

Next, we sought to categorize these dysregulated lncRNAs in all three cell types based on annotated human IncRNAs in the RefSeq database. In PASMCs, among antisense lncRNAs, there were 31 introns, 53 exons, 61 intergenic downstream, and 23 intergenic_upstream sequences (Figure $2 A$ ). Among sense lncRNAs, there were 24 introns, 18 exons, 22 intergenic_downstream, and 13 intergenic upstream sequences (Figure 2B). The largest proportion of sequences was antisense intergenic_upstream, which indicates the potential importance of this lncRNA.

Similarly, we categorized lncRNAs in PMECs based on the annotated human IncRNA sequences in the RefSeq database. Among the antisense lncRNAs, there were 58 introns, 76 exons, 31 intergenic_downstream, and 84 intergenic_upstream sequences (Figure 2C), while among sense lncRNAs, there were 26 introns, 25 exons, 

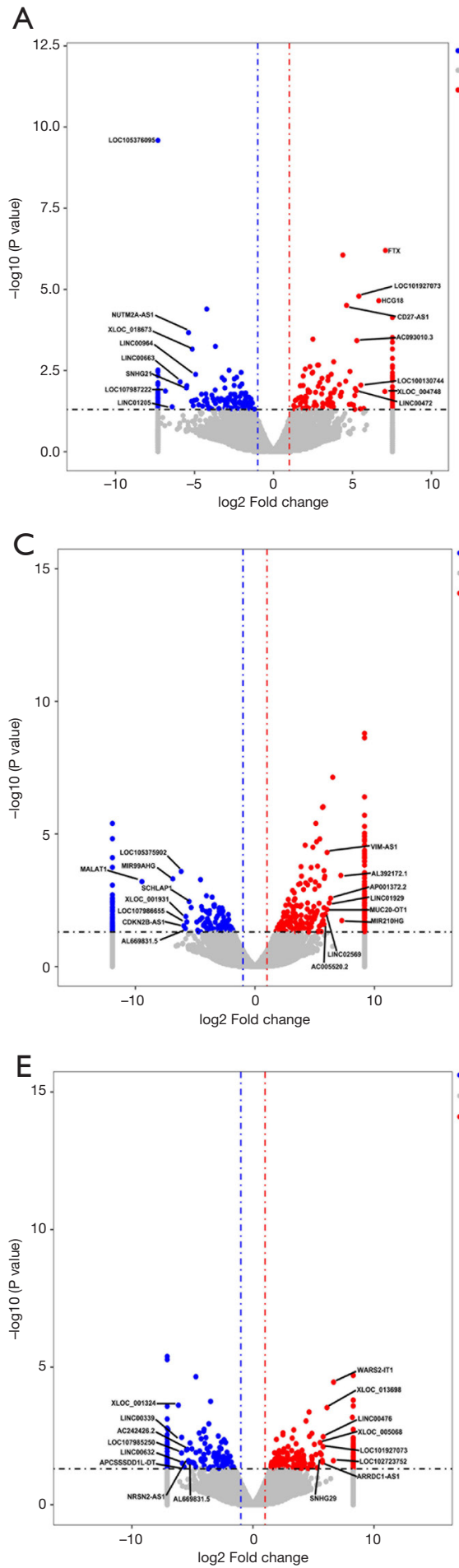

PASMCs

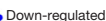

Not-significant

Up-regulated

B

PASMC H-vs.FASMC C: P value $<0.05^{\text {s8 }} \mid \log 2$ FCl $>1$

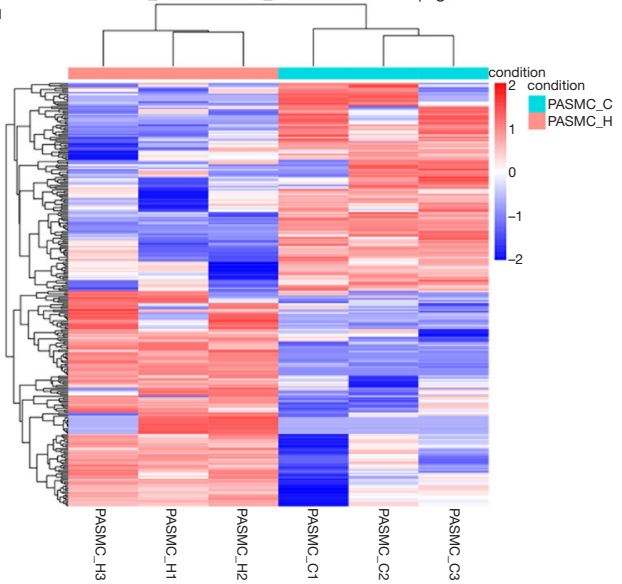

PMECs

- Down-regulated - Notsignificant -Up-regulated

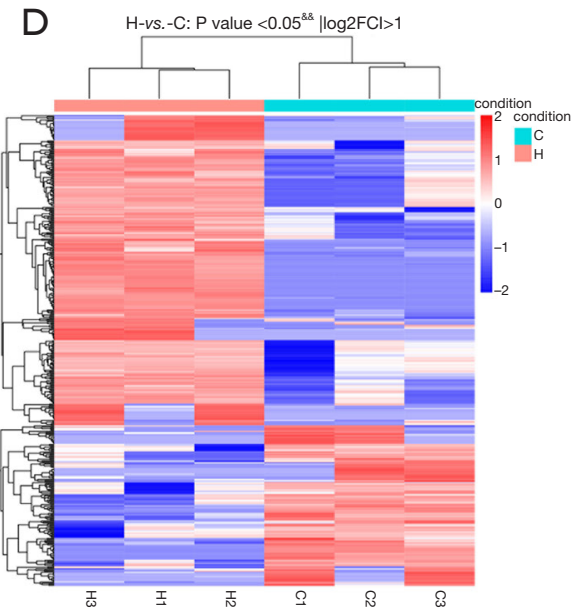

PCs

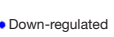
-Up-regulated

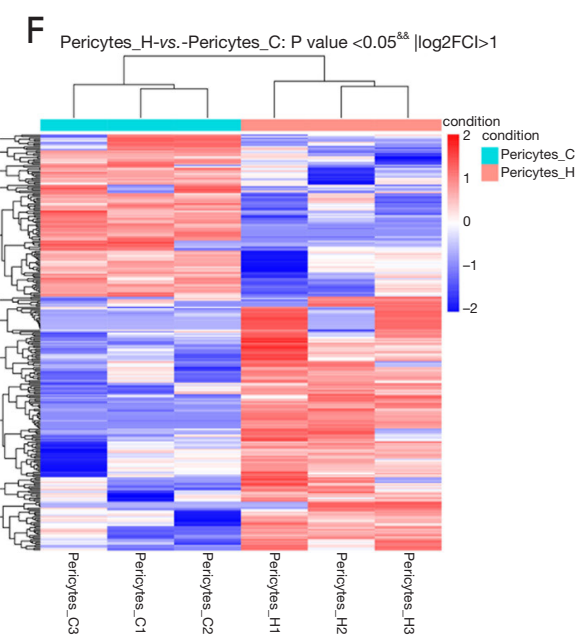

Figure 1 Overview of lncRNA profiles of pulmonary artery cells. (A,C,E) Volcano plot of the test group vs. the control group concerning lncRNAs of PASMCs, PMECs, and PCs. The blue vertical line corresponds to 2-fold down and the red vertical line corresponds to 2-fold up, while the horizontal line represents the $\mathrm{P}$ value of 0.05 . (B,D,F) Heatmap representing the expression distribution at single sample levels of the regulated genes in PASMCs, PMECs, and PCs. ${ }^{\& \&}, \mathrm{P}<0.05, \mathrm{H}$ vs. C. PASMCs, pulmonary artery smooth muscle cells; PMECs, pulmonary microvascular endothelial cells; PCs, pericytes. 


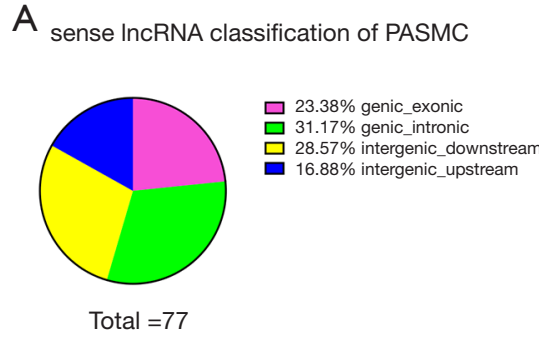

$\mathrm{D}_{\text {art }}$

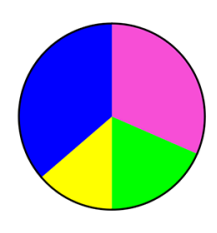

Total $=168$

Total $=77$

antisense IncRNA classification of PASMC

믄 $13.69 \%$ intergenic_downstream
B

sense IncRNA classification of PMEC

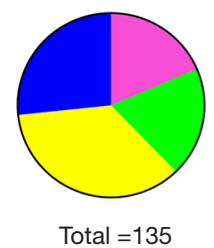

마. $18.52 \%$ genic_exonic

ㅁ $35.56 \%$ intergenic_downstream

$26.67 \%$ intergenic_upstream
C

sense IncRNA classification of PC

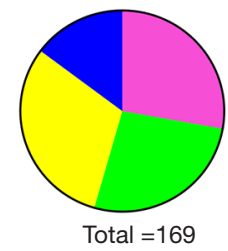

$\square 27.81 \%$ genic_exonic

$\square 26.63 \%$ genic_intronic

口 $30.77 \%$ intergenic_downstream

$\square 14.79 \%$ intergenic_upstream

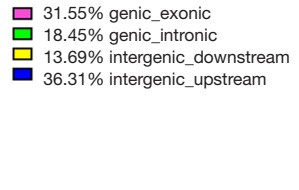

E

antisense IncRNA classification of PMEC

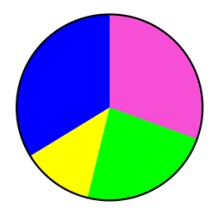

무을 genic_exonic

믄 $12.45 \%$ intergenic_downstream

- $33.73 \%$ intergenic_upstream
$\mathrm{F}$

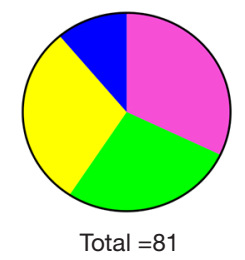

$32.10 \%$ genic_exonic

$29.63 \%$ intergenic downstream

$11.11 \%$ intergenic upstream

Figure 2 Overview of lncRNA profiles of pulmonary artery cells. (A,B,C) The category of antisense lncRNAs in PASMCs, PMECs, and PCs. (D,E,F) The category of sense lncRNAs in PASMCs, PMECs, and PCs. PASMCs, pulmonary artery smooth muscle cells; PMECs, pulmonary microvascular endothelial cells; PCs, pericytes.

48 intergenic_downstream, and 36 intergenic_upstream sequences (Figure 2D). Thus, the largest proportion was the antisense genic_exonic sequences, which again indicates the importance of this lncRNA.

In addition, we summarized the categories of these dysregulated lncRNAs in PCs based on the notes to human lncRNAs in the reference sequence database. Among antisense lncRNAs in PCs, there were 22 introns, 26 exons, 24 intergenic_downstream, and 9 intergenic_upstream sequences (Figure 2E), while among sense lncRNAs, there were 45 introns, 47 exons, 52 intergenic_downstream, and 25 intergenic_upstream sequences (Figure $2 F$ ). Clearly, the largest proportion was the antisense genic_exonic sequences, which may indicate the importance of this lncRNA.

\section{GO term analysis of differentially expressed lncRNAs in PASMCs, PMECs, and PCs}

To analyze the functions of these differentially expressed IncRNAs, GO term analyses showed the first 5 dysregulated processes in three paired groups (biological process, cellular component, and molecular function). The upregulated GO term enrichment of lncRNAs in PASMCs included negative regulators of cardiac muscle cell apoptotic processes, peripheral nervous system neuron development, vasculogenesis, zinc ion binding, and SNAP receptor activity (Figure $3 A$ ). In contrast, GO term enrichment of the downregulated lncRNAs included cellular process, negative regulators of intracellular transport, microtubule bundle formation, microtubuleassociated complex, and zinc ion/ATP/microtubule binding (Figure 3B).

GO term enrichment of the upregulated lncRNAs in PMECs included the HIF-1 $\alpha$ signaling pathway, the intrinsic apoptotic signaling pathway response to oxidative stress, and the microtubule-organizing center in PMECs (Figure 3C), while GO term enrichment of the downregulated lncRNAs included macrophage, microtubule-organizing center, and DNA/zinc ion/metal ion binding (Figure 3D).

GO term enrichment of the upregulated lncRNAs in PCs included heart morphogenesis, regulation of cell proliferation, positive regulation of apoptotic process, microtubule-organizing center, and microtubule/zinc ion binding (Figure $3 E$ ), while GO term enrichment of the downregulated lncRNAs included positive regulation of histamine secretion by mast cells, natural killer cell degranulation, eosinophil degranulation, and SNARE/zinc ion binding (Figure 3F). 

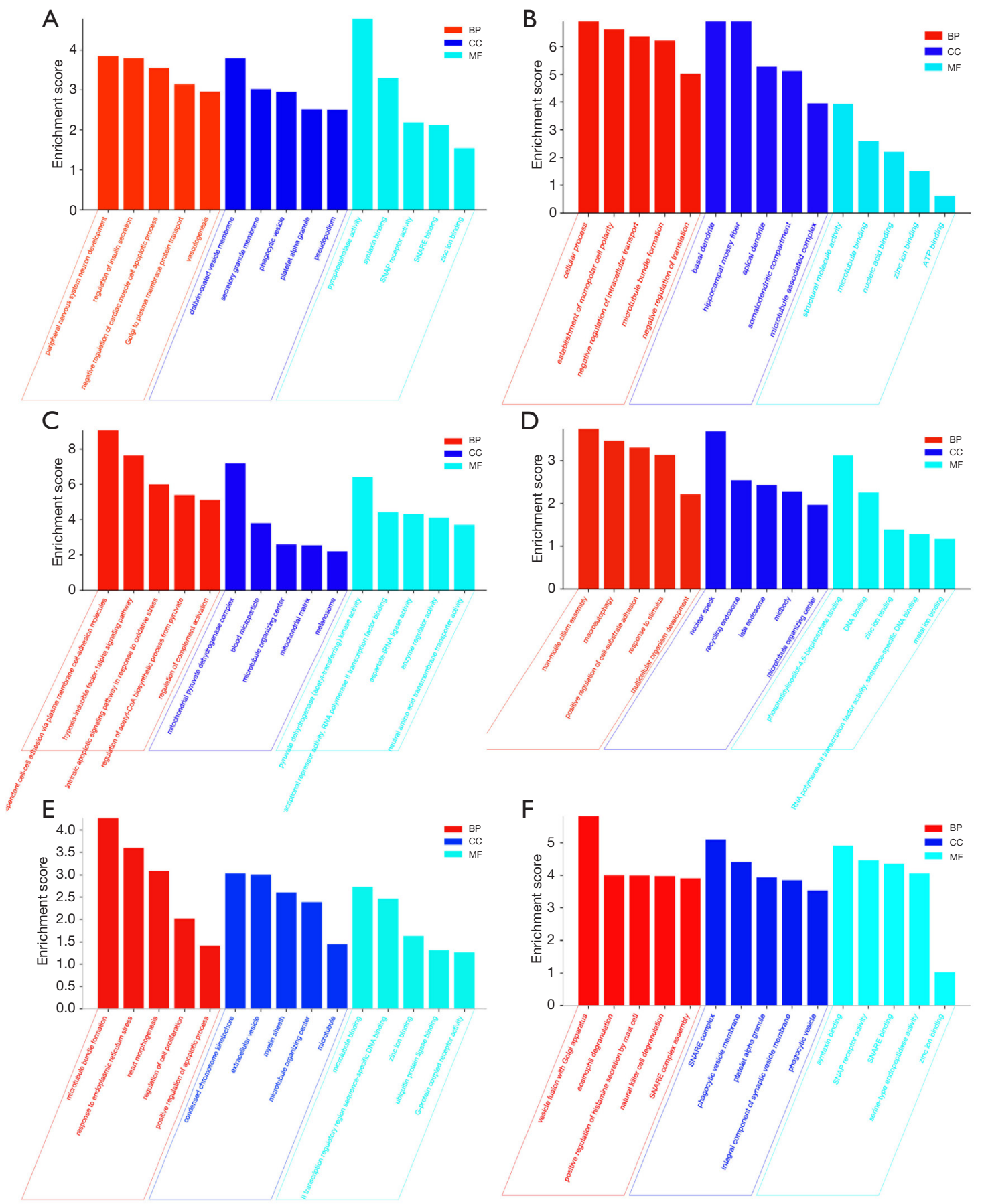

Figure 3 Gene Ontology for all dysregulated lncRNAs in PASMCs, PMECs, and PCs. (A,C,E) Top 5 GO terms of upregulated expressed lncRNAs of PASMCs, PMECs, and PCs were identified between the hypoxia and control samples. (B,D,F) Top 5 GO terms of downregulated expressed lncRNAs of PASMCs, PMECs, and PCs were identified between the hypoxia and control samples. BP, biological process; CC, cellular component; MF, molecular function; PASMCs, pulmonary artery smooth muscle cells; PMECs, pulmonary microvascular endothelial cells; PCs, pericytes. 

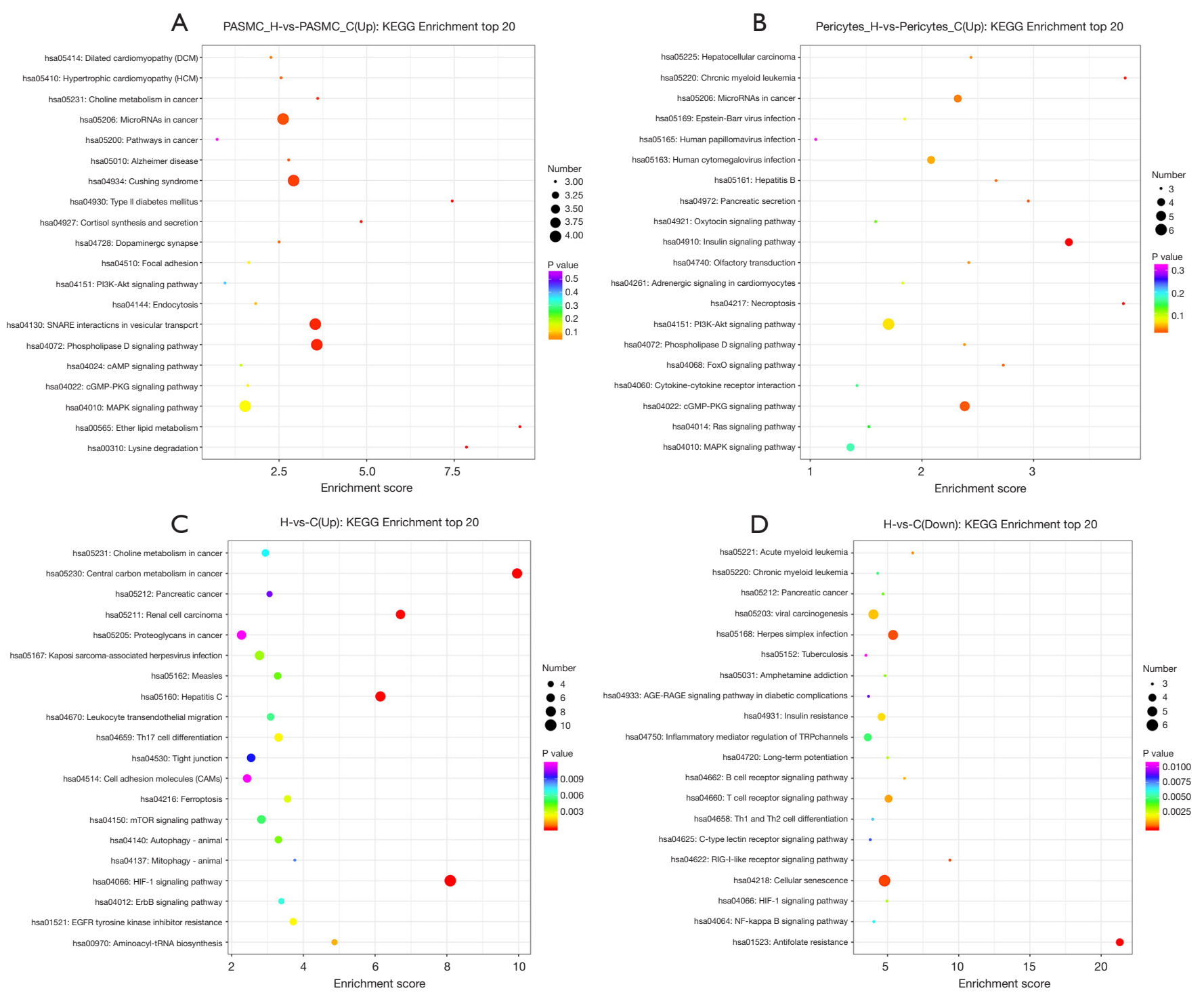

Figure 4 Kyoto Encyclopedia of Genes and Genomes pathway analyses for all dysregulated lncRNAs in PASMCs, PMECs, and PCs. (A,B,C) KEGG analysis of upregulated lncRNAs of PASMCs, PCs, and PMECs between the hypoxia and control samples. (D) KEGG analysis of downregulated lncRNAs of PMECs between the hypoxia and control samples. PASMCs, pulmonary artery smooth muscle cells; PMECs, pulmonary microvascular endothelial cells; PCs, pericytes.

\section{KEGG patbway enrichment analysis of differentially expressed lncRNAs in PASMCs, PMECs, and PCs}

KEGG pathway analysis showed that the upregulated lncRNAs in PASMCs were involved in 20 pathways, including microRNAs in cancer, the phospholipase D signaling pathway, the MAPK signaling pathway, hypertrophic cardiomyopathy (HCM), and dilated cardiomyopathy (DCM), among others (Figure 4A). The downregulated lncRNAs did not appear to be associated with any particular pathway.

Upregulated lncRNAs in PCs were also associated with 20 pathways, including the cGMP-PKG signaling pathway, the MAPK signaling pathway, microRNAs in cancer, the PI3K-Akt signaling pathway, and the insulin signaling pathway (Figure 4B), while the downregulated lncRNAs showed no significant involvement in any pathway.

Next, KEGG pathway analysis of the upregulated lncRNAs in PMECs also identified 20 pathways, and these 


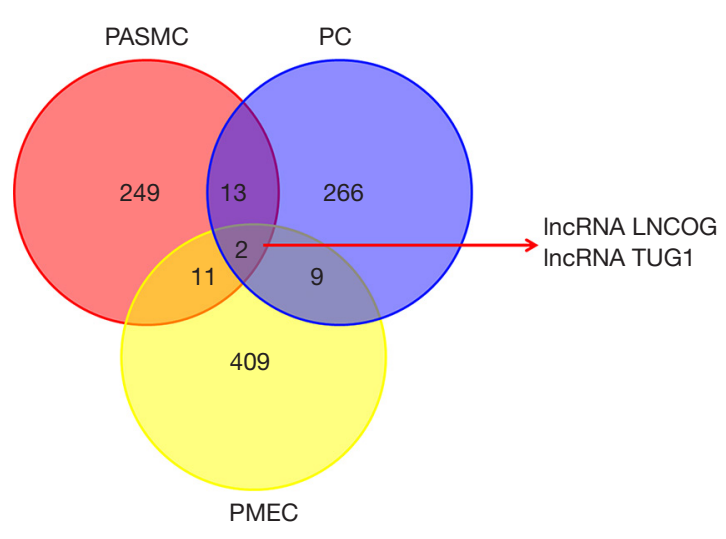

Figure 5 Overlapping Venn diagram of differentially expressed lncRNAs in PASMCs, PMECs, and PCs. PASMCs, pulmonary artery smooth muscle cells; PMECs, pulmonary microvascular endothelial cells; PCs, pericytes.

included the HIF-1 signaling pathway, the ErbB signal pathway, the mTOR signaling pathway, and autophagyanimal, among others (Figure 4C). In contrast to PASMCs, downregulated lncRNAs in PMECs were involved in 20 pathways, including cell senescence, the NF-kappa B signaling pathway, the HIF-1 signaling pathway, the T cell receptor signaling pathway, and viral carcinogenesis, among others (Figure 4D).

\section{Venn diagram for identifying overlapping but differentially expressed lncRNAs in PASMCs, PCs, and PMECs}

According to the screening criteria, 13 lncRNA sequences that were differentially expressed in both PASMCs and PMECs were identified. Among them, 2 lncRNAs were downregulated in both and 2 were upregulated, 2 lncRNAs were downregulated in PASMCs but upregulated in PMECs, and 6 were upregulated in PASMCs but downregulated in PMECs. Similarly, PASMCs and PCs shared 15 differentially expressed lncRNAs, where 5 lncRNAs were upregulated in both, 9 were downregulated in PASMCs but upregulated in PCs, and 1 was upregulated in PASMCs but downregulated in PCs. There were 11 shared but differentially expressed lncRNAs between PMECs and PCs. Of these, 5 were upregulated in both, 1 was downregulated in both, 3 were downregulated in PMECs but upregulated in PCs, and 2 lncRNAs were upregulated in PMECs but downregulated in PCs. The lncRNA LNCOG and the lncRNA TUG1 showed obvious differential expression in PASMCs, PMECs, and PCs
(Figure 5).

\section{Validation of $\operatorname{lncRNA}$ expression}

To verify data on differential expression, IncRNA TUG1 and lncRNA LNCOG were selected as representative genes for further validation by real-time PCR. The expression of these dysregulated lncRNAs was investigated in all 3 cell types, and real-time PCR results showed that lncRNA TUG1 expression was consistent with that obtained using sequencing data, suggesting the high reliability of sequencing. The real-time PCR results of lncRNA LNCOG were contrary to the sequencing results, and the underlying reasons for such a discrepancy need further exploration (Figure $6 A, B, C$, all $\mathrm{P}<0.05$ ). Specifically, lncRNA TUG1 was upregulated in PASMCs and PCs but downregulated in PMECs (Figure 6D,E,F, all $\mathrm{P}<0.05$ ). Heatmap of lncRNA LNCOG and lncRNA TUG1 in hypoxia-induced PASMCs, PMECs, and PCs are shown in Figure 6G,H,I.

\section{Annotation for lncRNA TUG1/miRNA/mRNA interaction}

According to reports, existing data suggest that lncRNAs act as miRNA sponges to regulate gene expression and further participate in the regulation of cell proliferation or apoptosis. Therefore, using the software LncRNA2 Target (v2.0), we hypothesized that the following miRNAs might bind to lncRNA TUG1, namely miR-129-5p, miR-204-5p, miR-138-5p, miR-335-5p, miR-9-5p, miR-34a -5p, miR145-5p, miR-153, miR-142-3p, miR-299-3p, and miR-300. Next, we used 3 databases, miRanda, microT, and PicTar, to predict target mRNAs of miR-129-5p, miR-138-5p, and miR-145-5p. We selected 10 mRNAs for miR-129-5p, 10 mRNAs for miR-138-5p, and 10 mRNAs for miR-145-5p (Figure 7A). To summarize, we speculated the mechanism of lncRNA TUG1 in pulmonary artery cells, which is shown in Figure $7 B$.

\section{Discussion}

To the best of our knowledge, this is the first description of the differential expression of lncRNAs in PASMCs, PMECs, and PCs under hypoxic conditions. However, there were significant differences in lncRNA TUG1 expression among the three types of cells, and the trends were not consistent. LncRNA TUG1 was upregulated in PASMCs and PCs, but downregulated in PMECs. 
A

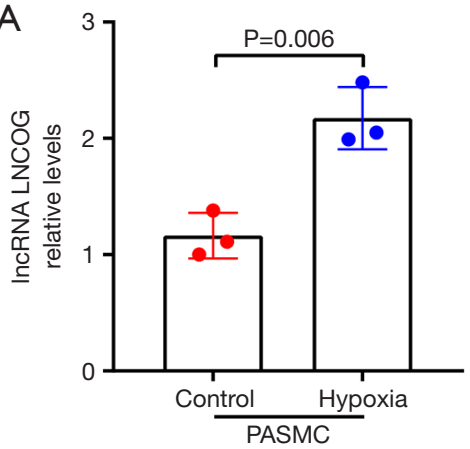

G

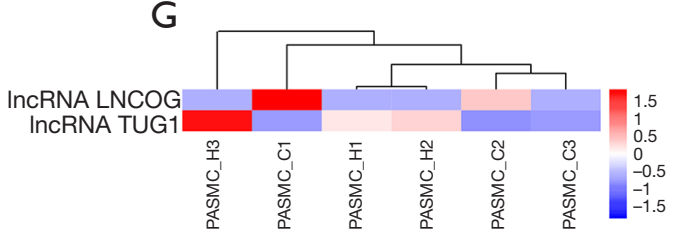

D

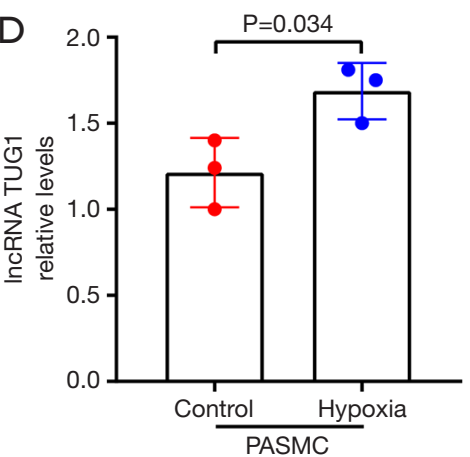

B

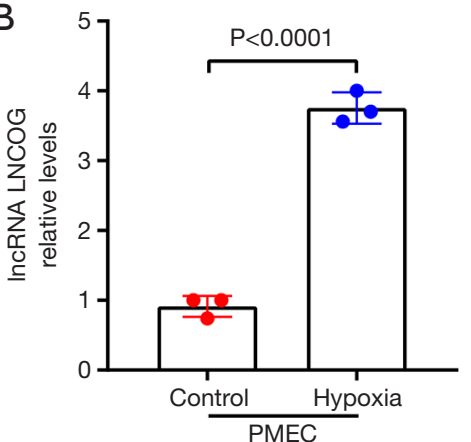

$\mathrm{H}$

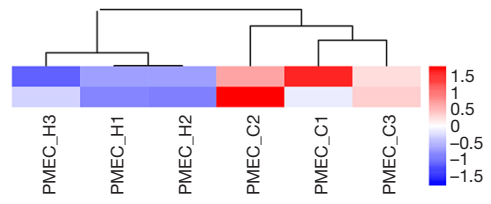

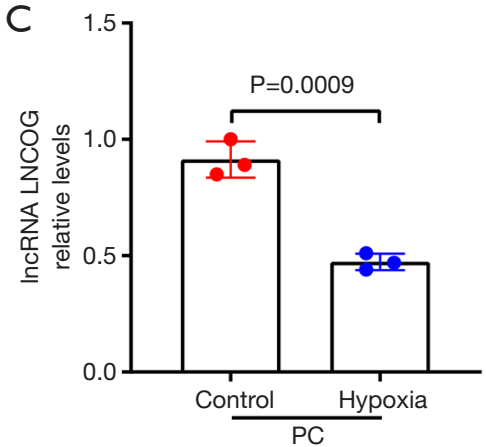

I

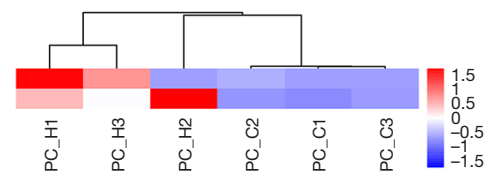

$\mathrm{F}$

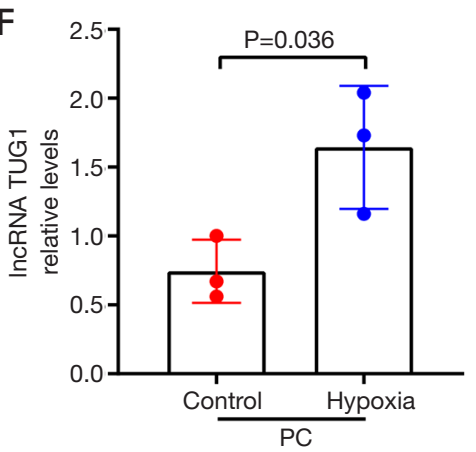

Figure 6 Validation of lncRNA expression. (A,B,C) LncRNA LNCOG validated by real-time PCR. (D,E,F) LncRNA TUG1 validated by real-time PCR. (G,H,I) Heatmap representing the expression distribution of lncRNA LNCOG and lncRNA TUG1 in PASMCs, PMECs, and PCs. PASMCs, pulmonary artery smooth muscle cells; PMECs, pulmonary microvascular endothelial cells; PCs, pericytes.

$\mathrm{PH}$ has become a public health problem that seriously threatens human physical and mental health (11). Accumulating research on the pathogenesis of HPH, along with the emergence of data from high-throughput sequencing, biochemical, and computational biology methods, has revealed that several types of non-coding RNAs, such as lncRNAs and miRNAs, participate in the pathophysiological processes underlying $\mathrm{PH}$ development, and that some of these molecules represent potential diagnostic or therapeutic targets $(12,13)$. Although currently reported in PASMCs and PMECs, and there are no reports on differential lncRNA expression and function of the three types of pulmonary artery cells together. Specifically, pertinent data on PCs has never been reported, and we show extensive differential expression of lncRNAs in PCs. It has been previously reported that IncRNA
MEG3 is downregulated in hypoxic PASMCs, but that lncRNA H19 is highly expressed in the lungs of rats/mice with monocrotaline-induced $\mathrm{PH}$, which can promote the proliferation of PASMCs (14-16). This trend is consistent with our sequencing results. We also did not find any differential expression in other reported lncRNAs. We think that there may be several reasons for this discrepancy. First, there are relatively few studies about lncRNAs in $\mathrm{PH}$, and second, differences in cells may also contribute to these discrepancies, and last, different anoxia method used between in the present study and the previous study may one reason leading to this discrepancy (3).

Next, GO analysis showed that processes such as cell proliferation and positive regulation of apoptotic process are associated with the abnormal proliferation of PASMCs and PMECs during the pathogenesis of $\mathrm{PH}$ (17). Furthermore, 

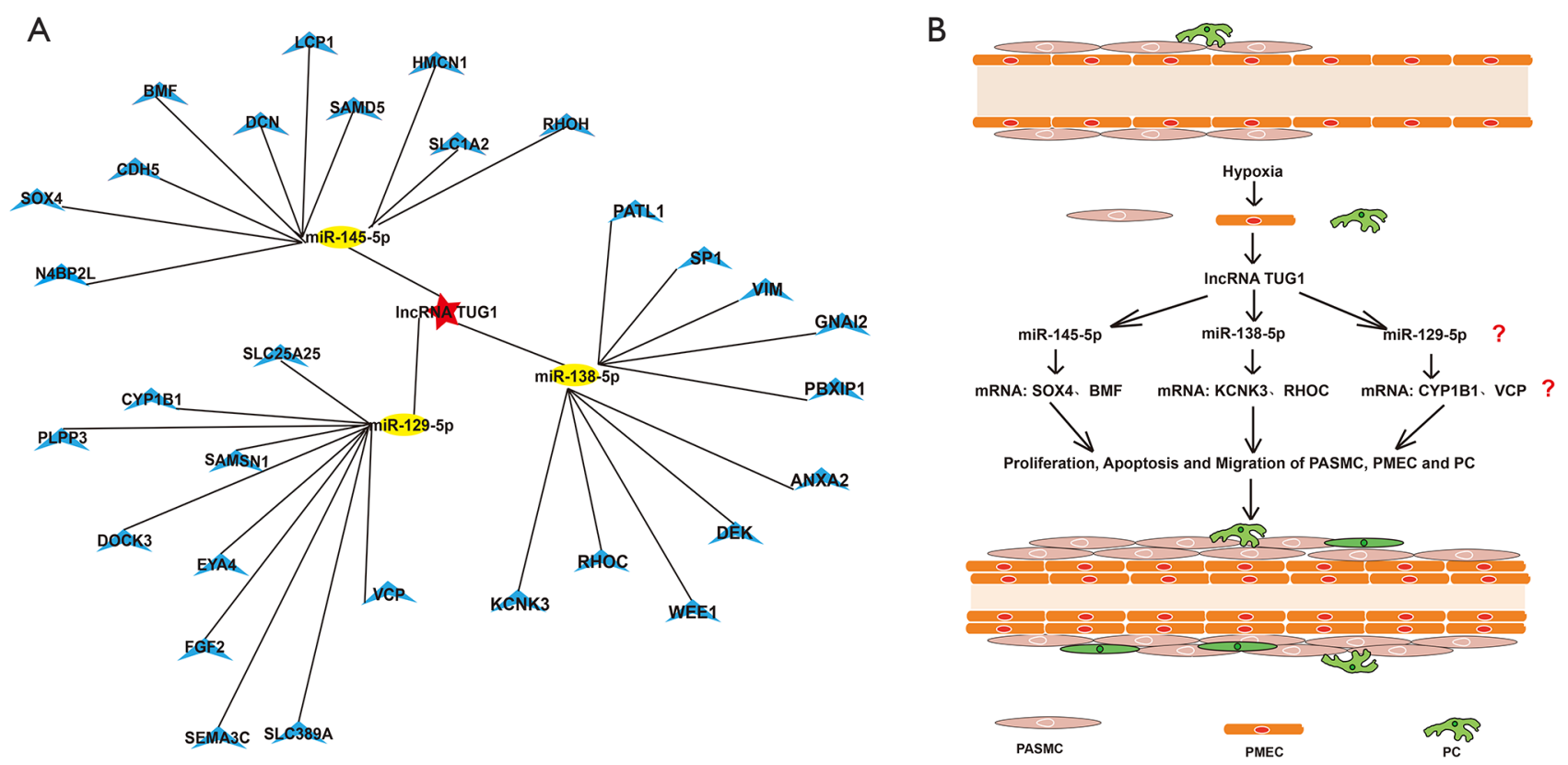

Figure 7 Predicted lncRNA-miRNA-mRNA relationships. (A) Blue represent mRNAs, yellow represent miRNAs, and the red represents the lncRNA. (B) Action mechanism diagram of lncRNA TUG1 in pulmonary artery cells. PASMCs, pulmonary artery smooth muscle cells; PMECs, pulmonary microvascular endothelial cells; PCs, pericytes.

KEGG pathway analysis also showed that multiple pathways, including the MAPK, CGMP-PKG, HIF-1, and PI3K-Akt signaling pathways, were significantly enriched among the upregulated lncRNAs. Notably, several studies found that knocking out lncRNA TCONS_00034812 promoted proliferation and inhibited apoptosis of PASMCs through several above-mentioned signaling pathways in $\mathrm{PH}$ (18-20).

LncRNA MEG3 was downregulated in hypoxic PASMCs (15), while lncRNA TUG1 was highly expressed in HPH mice and HPH-PASMCs (21). Our results show that lncRNA TUG1 is upregulated in PASMCs and PCs but downregulated in PMECs, and that it plays an important role in hypoxia cellular models. Other lncRNAs have not been studied in-depth and their specific roles remain to be elucidated. Moreover, despite numerous studies on differentially expressed lncRNAs in $\mathrm{PH}$, many challenges continue to exist with respect to accurate normalization of lncRNA levels for clinical use among various experiments. Nonetheless, our results indicate the possibility that lncRNAs are likely to participate in the pathogenesis of $\mathrm{PH}$.

Of all the differentially expressed lncRNAs in PASMCs, PMECs, and PCs, 2 of them, namely lncRNA TUG1 and lncRNA LOCNG, were differentially regulated in all three cell types. Available literature shows that lncRNA TUG1 is highly expressed in HPH-PASMCs (21), and this is consistent with our results. Notably, lncRNA TUG1 was downregulated in hypoxia-induced PMECs but upregulated in PCs. Its role in PMECs and PCs and its mechanism in pulmonary artery cells need to be further explored.

Growing evidence indicates that a large portion of lncRNAs can serve as miRNA "sponges", as they share common miRNA response elements and can influence posttranscriptional regulation by inhibiting available miRNA activity (22). Su et al. (14) have demonstrated that lncRNA H19 is highly expressed in MCT-induced rodent lungs and that it is upregulated in PDGF-BB-induced PASMCs. The lncRNA H19-let-7b-AT1R axis contributes to the pathogenesis of $\mathrm{PH}$ by stimulating PASMC proliferation. It has also been reported that lncRNA TUG1 is highly expressed in HPH-PASMCs and that silencing of lncRNA TUG1 downregulates the expression of Foxc1 by binding to $\mathrm{miR}-374 \mathrm{c}$ to inhibit the proliferation and migration of PASMCs, promoting PASMC apoptosis in HPH (21). Real-time PCR results showed that IncRNA TUG1 was significantly upregulated in PASMCs and PCs but downregulated in PMECs, and this is consistent with previous results. Therefore, we predicted miRNA partners 
of lncRNA TUG1 and verified this in different cells. However, the mechanistic aspects of its role in pulmonary artery cells need further evaluation.

The target gene miR-145-5p, predicted to interact with lncRNA TUG1, has been studied in PH. Specifically, miR$145-5 \mathrm{p}$ is upregulated in pulmonary arterial hypertension $(\mathrm{PAH})$ patients under hypoxic conditions in PASMCs, and in hypoxia-induced PAH rats. Furthermore, it can promote the proliferation and migration of hypoxia-induced PASMCs and prevent hypoxia-induced $\mathrm{PH}$ and pulmonary vascular remodeling (23). Lastly, for target genes miR$145-5 \mathrm{p}$ and $\mathrm{miR}-129-5 \mathrm{p}$, miR-129-5p is downregulated in damaged cardiomyocytes, but its overexpression can reduce cardiomyocyte apoptosis (24).

\section{Conclusions}

Our study, for the first time, describes the comprehensive expression profiles of differentially expressed lncRNAs in PASMCs, PMECs, and PCs when subjected to hypoxia. Among them, lncRNA TUG1 showed significant upregulation in PASMCs and PCs, but was downregulated in PMECs, which indicated that lncRNA TUG1 could regulate the function of pulmonary artery cells.

\section{Acknowledgments}

Funding: This study was supported by the Program of National Natural Science Foundation of China (81870042 and 81900050), National Science and Technology Information System of the People's Republic of China (2018YFC1313603), and Program of Natural Science Foundation of Shanghai (18ZR1431500), and Program of Shanghai Municipal Commission of Health (20204Y0382).

\section{Footnote}

Reporting Checklist: The authors have completed the MDAR reporting checklist. Available at http://dx.doi.org/10.21037/ atm-21-2040

Data Sharing Statement: Available at http://dx.doi. org/10.21037/atm-21-2040

Conflicts of Interest: All authors have completed the ICMJE uniform disclosure form (available at http://dx.doi. org/10.21037/atm-21-2040). The authors have no conflicts of interest to declare.
Ethical Statement: The authors are accountable for all aspects of the work in ensuring that questions related to the accuracy or integrity of any part of the work are appropriately investigated and resolved. All procedures followed were in accordance with the ethical standards of the responsible committee on human experimentation (institutional and national) and with the Helsinki Declaration (as revised in 2013). Shanghai Pulmonary Hospital reviewed and approved the protocol form prior to initiation of the study (approval number, K20-195Y).

Open Access Statement: This is an Open Access article distributed in accordance with the Creative Commons Attribution-NonCommercial-NoDerivs 4.0 International License (CC BY-NC-ND 4.0), which permits the noncommercial replication and distribution of the article with the strict proviso that no changes or edits are made and the original work is properly cited (including links to both the formal publication through the relevant DOI and the license). See: https://creativecommons.org/licenses/by-nc-nd/4.0/.

\section{References}

1. Kemdem A, Lemaitre F, Lovat R, et al. Acute hypoxic pulmonary hypertension associated with right heart failure. Acta Cardiol 2020;75:544-8.

2. Brock M, Schuoler C, Leuenberger C, et al. Analysis of hypoxia-induced noncoding RNAs reveals metastasisassociated lung adenocarcinoma transcript 1 as an important regulator of vascular smooth muscle cell proliferation. Exp Biol Med (Maywood) 2017;242:487-96.

3. Bischoff FC, Werner A, John D, et al. Identification and Functional Characterization of Hypoxia-Induced Endoplasmic Reticulum Stress Regulating lncRNA (HypERlnc) in Pericytes. Circ Res 2017;121:368-75.

4. Li WW, Cao AH, Sun FY. LncRNA MIAT stimulates oxidative stress in the hypoxic pulmonary hypertension model by sponging miR-29a-5p and inhibiting Nrf2 pathway. Eur Rev Med Pharmacol Sci 2020;24:9022-9.

5. Ma Y, Zhang JM, Wen LX, et al. Membrane-lipid associated lncRNA: A new regulator in cancer signaling. Cancer Lett 2018;419:27-9.

6. Zahid KR, Raza U, Chen J, et al. Pathobiology of pulmonary artery hypertension: role of long non-coding RNAs. Cardiovasc Res 2020;116:1937-47.

7. Wu G, Cai J, Han Y, et al. LincRNA-p21 regulates neointima formation, vascular smooth muscle cell proliferation, apoptosis, and atherosclerosis by enhancing 
p53 activity. Circulation 2014;130:1452-65.

8. Josipovic I, Fork C, Preussner J, et al. PAFAH1B1 and the lncRNA NONHSAT073641 maintain an angiogenic phenotype in human endothelial cells. Acta Physiol (Oxf) 2016;218:13-27.

9. Langmead B, Salzberg S L. Fast gapped-read alignment with Bowtie 2. Nat Methods 2012;9:357-9.

10. Yuan $\mathrm{P}, \mathrm{Wu} W H$, Gong SG, et al. Impact of circGSAP in Peripheral Blood Mononuclear Cells on Idiopathic Pulmonary Arterial Hypertension. Am J Respir Crit Care Med 2021. [Epub ahead of print]. doi: 10.1164/ rccm.202005-2052LE.

11. Hansmann G, Koestenberger M, Alastalo TP, et al. 2019 updated consensus statement on the diagnosis and treatment of pediatric pulmonary hypertension: The European Pediatric Pulmonary Vascular Disease Network (EPPVDN), endorsed by AEPC, ESPR and ISHLT. J Heart Lung Transplant 2019;38:879-901.

12. Bienertova-Vasku J, Novak J, Vasku A. MicroRNAs in pulmonary arterial hypertension: pathogenesis, diagnosis and treatment. J Am Soc Hypertens 2015;9:221-34.

13. Deng L, Bradshaw AC, Baker AH. Role of noncoding RNA in vascular remodelling. Curr Opin Lipidol 2016;27:439-48.

14. Su H, Xu X, Yan C, et al. LncRNA H19 promotes the proliferation of pulmonary artery smooth muscle cells through AT1R via sponging let-7b in monocrotalineinduced pulmonary arterial hypertension. Respir Res 2018;19:254-72.

15. Zhu B, Gong Y, Yan G, et al. Down-regulation of lncRNA MEG3 promotes hypoxia-induced human pulmonary artery smooth muscle cell proliferation and migration via repressing PTEN by sponging miR-21. Biochem Biophys Res Commun 2018;495:2125-32.

16. Omura J, Habbout K, Shimauchi T, et al. Identification of Long Noncoding RNA H19 as a New Biomarker and Therapeutic Target in Right Ventricular Failure in Pulmonary Arterial Hypertension. Circulation

Cite this article as: $\mathrm{Lv} \mathrm{Z}$, Jiang $\mathrm{R}, \mathrm{Hu} \mathrm{X}$, Zhao Q, Sun Y, Wang L, Li J, Miao Y, Wu W, Yuan P. Dysregulated lncRNA TUG1 in different pulmonary artery cells under hypoxia. Ann Transl Med 2021;9(10):879. doi: 10.21037/atm-21-2040
2020;142:1464-84.

17. Dong F, Zhang J. Carboxyl terminus of Hsc70-interacting protein (CHIP) promotes pulmonary artery smooth muscle cell (PASMC) proliferation via enhancement of intracellular $\mathrm{Ca}(2+)$ concentration $([\mathrm{Ca}(2+)] \mathrm{i})$. Experimental Lung Research 2020;46:332-40.

18. Zhang Z, Li Z, Wang Y, et al. Overexpressed long noncoding RNA CPS1-IT alleviates pulmonary arterial hypertension in obstructive sleep apnea by reducing interleukin-1beta expression via HIF1 transcriptional activity. J Cell Physiol 2019;234:19715-27.

19. Zhu TT, Sun RL, Yin YL, et al. Long noncoding RNA UCA1 promotes the proliferation of hypoxic human pulmonary artery smooth muscle cells. Pflugers Arch 2019;471:347-55.

20. Wang YY, Cheng XD, Jiang H. Effect of atorvastatin on pulmonary arterial hypertension in rats through PI3K/ AKT signaling pathway. Eur Rev Med Pharmacol Sci 2019;23:10549-56.

21. Yang L, Liang H, Shen L, et al. LncRNA Tug1 involves in the pulmonary vascular remodeling in mice with hypoxic pulmonary hypertension via the microRNA-374cmediated Foxc1. Life Sci 2019;237:116769-802.

22. Zhuo Y, Zeng Q, Zhang P, et al. Functional polymorphism of lncRNA MALAT1 contributes to pulmonary arterial hypertension susceptibility in Chinese people. Clin Chem Lab Med 2017;5 5:38-46.

23. Yue Y, Zhang Z, Chen S, et al. miR-143 and miR-145 promote hypoxia-induced proliferation and migration of pulmonary arterial smooth muscle cells through regulating ABCA1 expression. Cardiovasc Pathol 2018;37:15-25.

24. Chen ZX, He D, Mo QW, et al. MiR-129-5p protects against myocardial ischemia-reperfusion injury via targeting HMGB1. Eur Rev Med Pharmacol Sci 2020;24:4440-50.

(English Language Editor: C. Betlazar-Maseh) 